\title{
Pulmonary artery dilatation in a young man presenting with a left mandibular fracture: Do not forget cocaine
}

\author{
Kohmal Ashok Solanki, Zhan Yun Lim, Andrea Pisesky, John Hogan
}

\begin{abstract}
Introduction: The use of crack cocaine in the United Kingdom has risen dramatically over the last decade, leading to an increase in serious respiratory complications, which can often go undiagnosed for years. Here we report a case of a 29-year-old male admitted to the emergency department with a left mandibular fracture and chest pain after a cocaine binge and subsequent assault. Case Report: An abnormal chest radiograph taken as part of a trauma series led to further investigations elucidating the eventual diagnosis of pulmonary artery dilatation likely secondary to cocaine. Conclusion: To our knowledge, this is the first case reporting pulmonary artery dilatation secondary to cocaine in the absence of pulmonary hypertension. The patient was treated conservatively with outpatient right-heart catheterization and serial transthoracic echocardiography to monitor for emerging pulmonary hypertension. We briefly
\end{abstract}

Kohmal Ashok Solanki ${ }^{1}$, Zhan Yun Lim² ${ }^{2}$ Andrea Pisesky ${ }^{3}$, John Hogan 4

Affiliations: ${ }^{1}$ BSc MBBS, Cardiology Foundation Year 1 Doctor, Department of Cardiology, Whipps Cross Hospital, London, United Kingdom; ${ }^{2} \mathrm{MBBS}$ MRCP, Cardiology Specialty Trainee, Department of Cardiology, Whipps Cross Hospital, London, United Kingdom; ${ }^{3} \mathrm{MBBS}$, Cardiology Foundation Year 1 Doctor, Department of Cardiology, Whipps Cross Hospital, London, United Kingdom; ${ }^{4} \mathrm{BSC}$ (Hons), MBChB, MD, FRCP, Consultant Cardiologist, Department of Cardiology, Whipps Cross Hospital, London, United Kingdom.

Corresponding Author: Kohmal Ashok Solanki, Flat 21 Jenner House, Hunter Street, London, United Kingdom. WC1N 1BL; Ph: +447749111538; E-mail: kohmalsolanki@ yahoo.co.uk

Received: 20 March 2013

Accepted: 15 May 2013

Published: 01 October 2013 discuss pulmonary artery dilatation and the effects of cocaine on the pulmonary vasculature.

Keywords: Cocaine, Pulmonary artery dilatation

$$
* * * * * * * * *
$$

Solanki KA, Lim AY, Pisesky A, Hogan J. Pulmonary artery dilatation in a young man presenting with a left mandibular fracture: Do not forget cocaine. International Journal of Case Reports and Images 2013;4(10):559-562.

$* * * * * * * * *$

doi:10.5348/ijcri-2013-10-379-CR-8

\section{INTRODUCTION}

After cannabis, cocaine is the second most used illicit substance in Europe, with the United Kingdom having the highest prevalence of cocaine use among young adults [1]. It is known to cause numerous respiratory complications, usually from smoking its free-base or "crack" form [2]. This case illustrates the importance of a full systemic workup of patients with a history of cocaine use, regardless of their presentation. To our knowledge, this is the first case reporting pulmonary artery dilatation secondary to cocaine in the absence of pulmonary hypertension [3].

\section{CASE REPORT}

A 29-year-old male (by profession carpenter) presented with head injury and chest pain after found by the police on the street pavement following an assault. He reported facial and chest wall tenderness. He had been consuming spirits, smoking cannabis and 2-3 grams of crack cocaine weekly for the last six years. He had no past medical history and family history of note. He denied any other symptoms on systematic review. On examination, his Glasgow coma scale (GCS) was 15/15 
and he was hemodynamically stable. Examination of his cardiovascular, respiratory, abdominal and neurological systems was unremarkable. Facial examination demonstrated swelling and a step deformity of his left lower jaw as well as a subgaleal hematoma over his left fronto-parietal region. An abnormal chest radiograph taken as part of a trauma series necessitated medical review.

The electrocardiogram of patient showed partial right bundle branch block that was not dynamic. His full blood count, urea and electrolytes, liver function tests and clotting were normal. Autoantibody screen showed no evidence of connective tissue disease. The 12 hour troponin was also negative. Chest radiograph revealed an enlarged heart and widened mediastinum (Figure 1). Subsequent two-dimensional (2D) transthoracic echocardiography (TTE) excluded an intra-cardiac shunt and demonstrated a dilated pulmonary artery, normal right atrium (RA) and right ventricle (RV) (Figure 2A-C) and no significant pulmonary regurgitation (Figure 2D). There was no evidence of pulmonary hypertension. Computed tomography (CT) scan of the chest with contrast was organized to further visualize the mediastinum and the great arteries. This revealed a $4.5-\mathrm{cm}$ dilated pulmonary artery trunk (normal upper limit is $3.3 \mathrm{~cm}$ ) (Figure 3), as well as a shallow right-sided pneumothorax and bilateral airspace opacification at the lung bases compatible with fluid or blood within the airspaces [3]. There was no evidence of an intra- or extra-cardiac shunt on multi-slice CT scan.

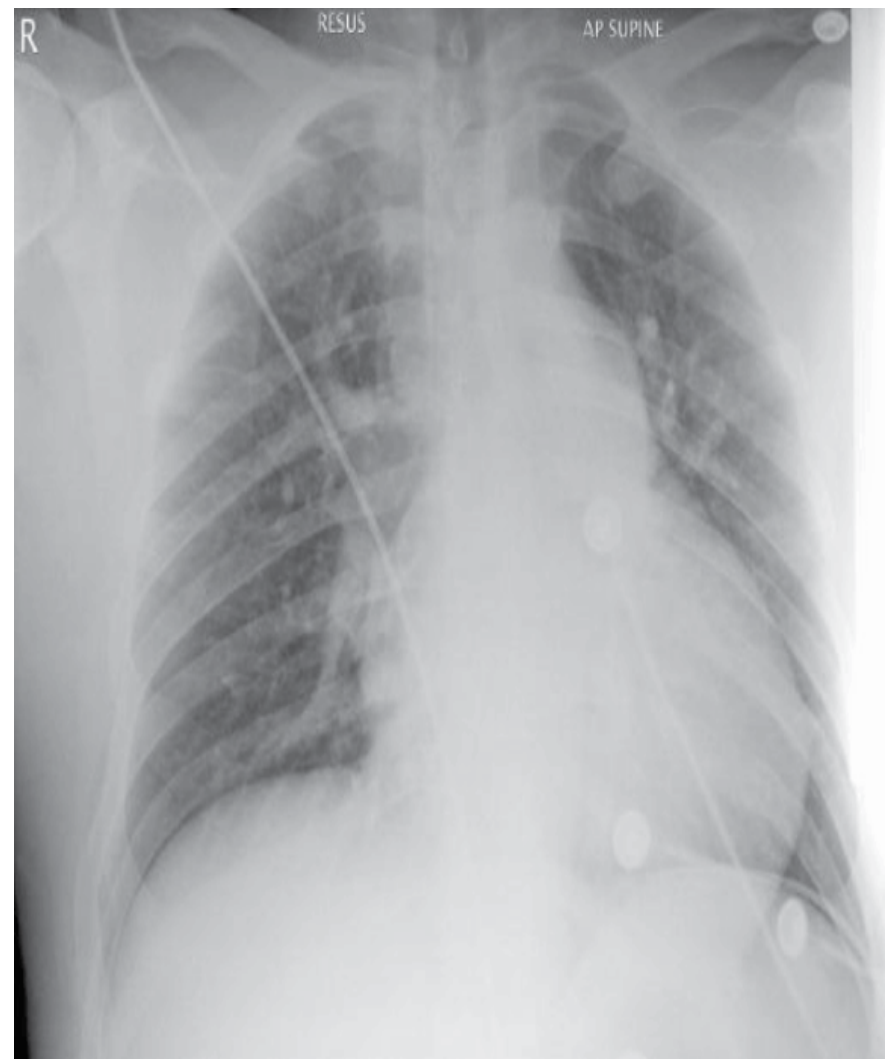

Figure 1: Chest radiograph demonstrating cardiomegaly, a widened mediastinum and increased pulmonary vasculature.
Pulmonary artery dilatation (PAD) is associated with congenital cardiovascular disease, systemic vasculitides, connective tissue disorders, infection, including trauma, and in rare cases labeled as idiopathic. In our case, patient denied any symptoms of chronic cardiovascular or respiratory disease nor known to have any systemic or hematological features of vasculitic or infectious disease. As part of the workup for the underlying aetiology, a connective tissue disease screen, TTE and a multi-slice CT scan of chest with contrast should be performed. A further right-heart cardiac catheterization should also be considered. The differential diagnosis at this stage would include cocaine-induced $\mathrm{PAD}$, although pulmonary hypertension would need to be formally excluded by right-heart catheterization even with normal pulmonary artery pressures on TTE in this patient.

The patient was managed conservatively due to the asymptomatic long-term history and lack of evidence of pulmonary hypertension. The patient was discharged with a view to formally assessing for pulmonary hypertension with right-heart cardiac catheterization. The patient will be subsequently followed-up up with serial TTEs every 6-12 months to assess if any evidence of pulmonary hypertension develops.

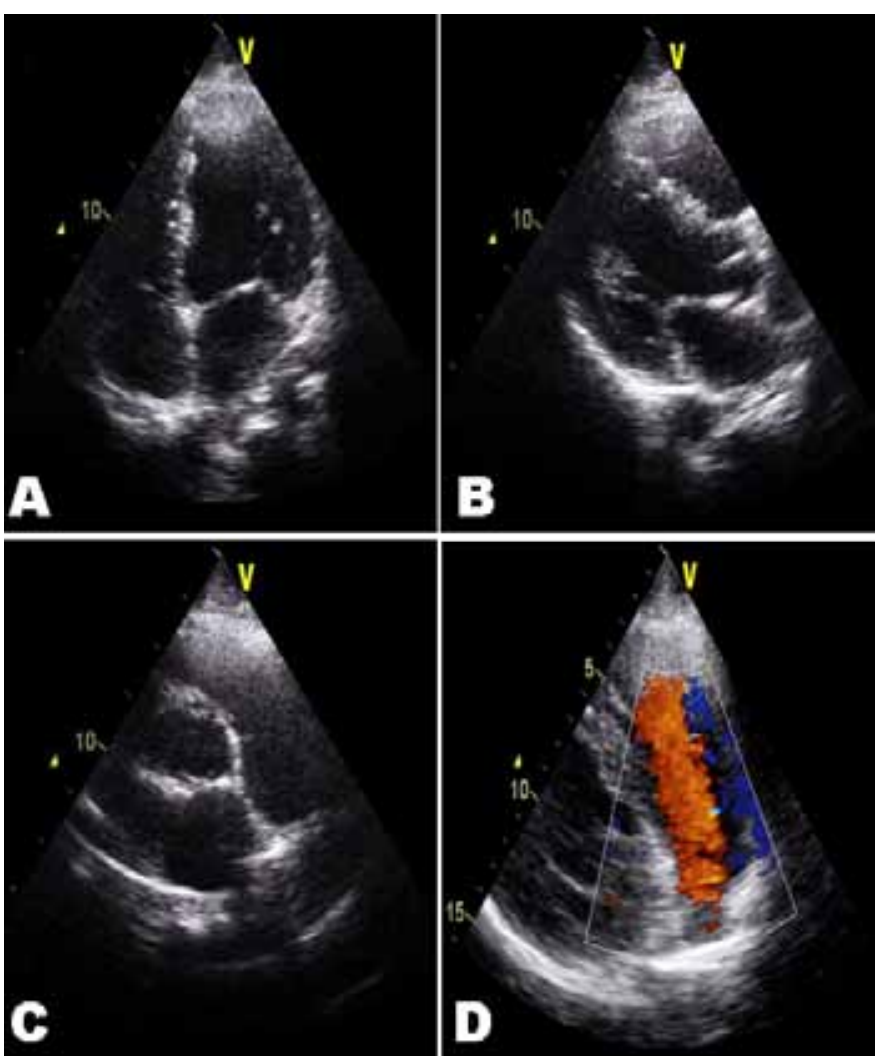

Figure 2: 2D echocardiography views: (A) apical four chamber view, (B) parasternal long axis view both showing a non-dilated right atrium and right ventricle, $(C)$ parasternal short axis view demonstrating an enlarged pulmonary artery, (D) Doppler echo showing no significant pulmonary regurgitation. 


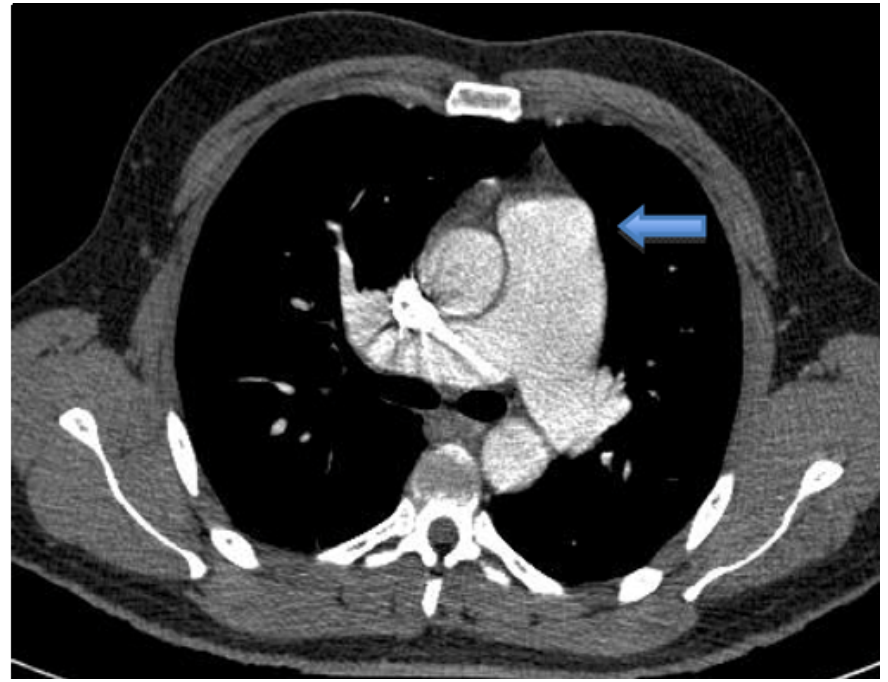

Figure 3: Contrast-enhanced computed tomography of the chest demonstrating a $4.5 \mathrm{~cm}$ dilated main pulmonary artery (blue arrow) extending into the right and left pulmonary arteries.

\section{DISCUSSION}

Cocaine is a naturally occurring alkaloid, that when extracted from the leaf of the Erythroxylon coca plant forms cocaine hydrochloride. However, due to its high melting point, it is unable to be smoked in this form. Cocaine hydrochloride can be transformed into its alkaloid form as either free-base (dissolved in water, then adding a base and a solvent) or crack (dissolved in water with sodium bicarbonate) cocaine which is more stable and thus suitable for smoking [4]. Crack cocaine reaches the cerebral circulation in less than half the time taken by the intravenous route, and 30-50 times faster than by nasal insufflation [2], thus making it the route of choice amongst most cocaine users. Cocaine is often cut with adulterants including bulking agents (e.g., talc, lactose or mannitol), stimulants (e.g., caffeine) and local anaesthetics (e.g., lidocaine) [4], inevitably leading to further systemic sequelae.

The pulmonary complications of either free-base or crack cocaine are numerous and have been well documented in literature $[2,5,6]$. Cocaine mediates its local anaesthetic effects by inhibiting sodium channels in neurons, thus blocking nerve conduction. It also acts as a sympathomimetic by inhibiting central and peripheral neuronal catecholamine uptake by adrenergic receptor stimulation [7]. Alpha-adrenoceptors in the pulmonary vasculature have been implicated in cocaine-induced pulmonary hypertension by pulmonary vasoconstriction [8]. However, intravenous cocaine hydrochloride was not found to cause short-term increases in pulmonary artery pressure or stroke volume, suggesting that there may be other contributory pathophysiological mechanisms [9]. Significant abnormalities in the pulmonary vasculature have also been associated with foreign additives in cocaine preparations such as talc, silica and lactose, causing granulomatous obstruction and microembolization [5, 10]. However, pulmonary artery medial hypertrophy was also found in cocaine users without foreign particle microembolisation [11], giving further credence to the pharmacological hypothesis that cocaine itself has a direct effect on the pulmonary vasculature.

Chronic cocaine use is a rare cause of PAD. To our knowledge, this is the first report to characterise this in the absence of pulmonary hypertension. Providing its course remains asymptomatic without significant changes in size of the pulmonary artery, the condition is generally considered benign and patients can undergo a prolonged period of follow-up with surgical repair if necessary. This is further evidenced by previous case reports of patients with idiopathic PAD who have had lengthy follow-up periods of up to 46 years [12]. However, in the case we have presented, continuing use of cocaine by the patient may necessitate follow-up at more frequent intervals.

\section{CONCLUSION}

Patients with a history of cocaine use require a full, thorough systematic work-up regardless of their presentation. Pulmonary complications, including pulmonary artery dilatation, can occur asymptomatically in cocaine users. Pulmonary artery dilatation can occur in the absence of pulmonary hypertension on transthoracic echocardiography. Asymptomatic pulmonary artery dilatation can be managed conservatively with right heart cardiac catheterization and surveillance serial transthoracic echocardiography.

$$
* * * * * * * * *
$$

\section{Author Contributions}

Kohmal Ashok Solanki - Substantial contributions to conception and design, Acquisition of data, Drafting the article, Critical revision of the article, Final approval of the version to be published

Zhan Yun Lim - Substantial contributions to conception and design, Acquisition of data, Drafting the article, Critical revision of the article, Final approval of the version to be published

Andrea Pisesky - Substantial contributions to conception and design, Acquisition of data, Drafting the article, Final approval of the version to be published

John Hogan - Conception and design, Drafting the article, Critical revision of the article, Final approval of the version to be published

\section{Guarantor}

The corresponding author is the guarantor of submission.

\section{Conflict of Interest}

Authors declare no conflict of interest. 


\section{Copyright}

(C) Kohmal Ashok Solanki et al. 2013; This article is distributed under the terms of Creative Commons attribution 3.0 License which permits unrestricted use, distribution and reproduction in any means provided the original authors and original publisher are properly credited. (Please see www.ijcasereportsandimages.com/ copyright-policy.php for more information.)

\section{REFERENCES}

1. The EMCDDA annual report 2010: The state of the drugs problem in Europe. Euro surveillance: Bulletin europeen sur les maladies transmissibles [European communicable disease bulletin] 2010;15(46).

2. Haim DY, Lippmann ML, Goldberg SK, Walkenstein MD. The pulmonary complications of crack cocaine. A comprehensive review. Chest 1995 Jan;107(1):23340.

3. Edwards PD, Bull RK, Coulden R. CT measurement of main pulmonary artery diameter. Br J Radiol 1998 Oct;71(850):1018-20.

4. Warner EA. Cocaine abuse. Ann Intern Med 1993 Aug 1;119(3):226-35.
5. Restrepo CS, Carrillo JA, Martinez S, Ojeda P, Rivera AL, Hatta A. Pulmonary complications from cocaine and cocaine-based substances: Imaging manifestations. Radiographics 2007 JulAug;27(4):941-56.

6. Terra Filho M, Yen CC, Santos Ude P, Muñoz DR. Pulmonary alterations in cocaine users. Sao Paulo Med J 2004 Jan 8;122(1):26-31.

7. Billman GE. Cocaine: a review of its toxic actions on cardiac function. Crit Rev Toxicol 1995;25(2):113-32.

8. Salvi SS. Alpha1-adrenergic hypothesis for pulmonary hypertension. Chest 1999 Jun;115(6):1708-19.

9. Kleerup EC, Wong M, Marques-Magallanes JA, Goldman MD, Tashkin DP. Acute effects of intravenous cocaine on pulmonary artery pressure and cardiac index in habitual crack smokers. Chest 1997 Jan;111(1):30-5.

10. Tomashefski JF Jr, Hirsch CS. The pulmonary vascular lesions of intravenous drug abuse. Hum Pathol 1980 Mar;11(2):133-45.

11. Murray RJ, Smialek JE, Golle M, Albin RJ. Pulmonary artery medial hypertrophy in cocaine users without foreign particle microembolization. Chest 1989 Nov;96(5):1050-3.

12. Ring NJ, Marshall AJ. Idiopathic dilatation of the pulmonary artery. Br J Radiol 2002 Jun;75(894):5325 .
Access full text article on other devices

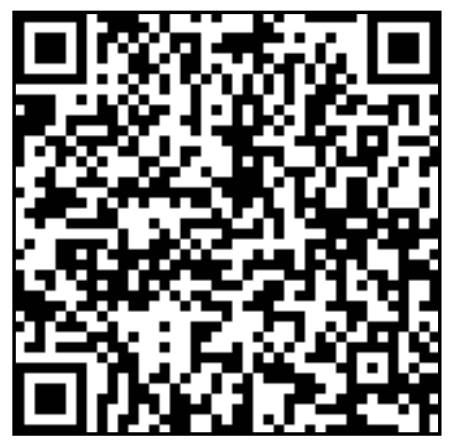

Access PDF of article on other devices

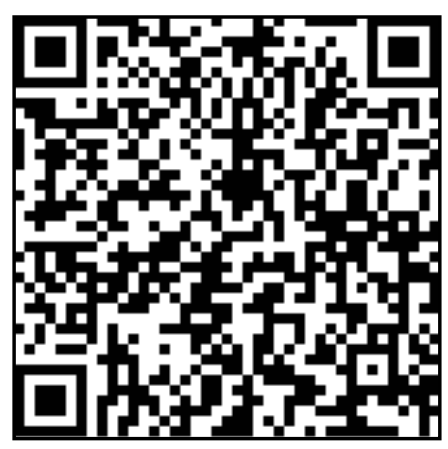

